\title{
Some aspects of saddlepoint approximations for non-parametric statistics
}

\author{
RUNGAO Jin
}

This thesis is concerned with Edgeworth and saddlepoint approximations and robust permutation tests in which the saddlepoint approximation has been used.

There are five chapters in this thesis. The first chapter is the introduction. The next three chapters are concerned with Edgeworth and saddlepoint approximations. The last chapter is about robust permutation tests in which the large deviation results related to the saddlepoint approximation have been used.

Chapter 2 contains saddlepoint approximations to the probability and probability density and tail probability of $\bar{X}$, the mean of a sample from a finite population. A unification of work based on the conditional saddlepoint and Edgeworth approximations is obtained although there are some original contributions in the details of the proof. In Section 1 of Chapter 2, the lattice situation is considered. In Section 2, the general case is considered.

In Chapter 3 the saddlepoint approximations near the endpoints of the support are obtained for binomial, hypergeometric, one and two sample Wilcoxon statistics. The usual saddlepoint approximations of Daniels (1954) are based on asymptotic normality of tilted means. However, if the means have finite support then the approximations can fail near the endpoints of the support where asymptotic normality of the tilted means does not hold. This chapter gives new accurate methods for saddlepoint approximations at the extremes of the support and new relative error rates are obtained near the endpoints for these statistics. The new approximations are compared numerically to those based on the normal saddlepoint method for one-sample and two-sample Wilcoxon statistcs.

In Chapter 4 a new saddlepoint approximation is obtained based on the exact moment generating function of van Dantzig (1947-1950). These approximations are compared numerically to those based on the conditional saddlepoint method and to the method of Froda and van Eeden [1] for $n=m=5$ and $m=10, n=6$. In general, we see the new method is a little better than the conditional method but not at all points,

Received 12th February, 2001

Thesis submitted to the University of Sydney, April 2000. Degree approved, October 2000. Supervisor: Professor John Robinson.

Copyright Clearance Centre, Inc. Serial-fee code: 0004-9727/01 \$A2.00+0.00. 
as expected since they have the same order of relative error. The method of Froda and van Eeden [1] is not effectively different in the compact region, but it starts to fail for large deviations and is quite poor near the endpoints of the support.

Chapter 5 considers the robust permutation tests in the one and two sample problems based on the $M$-estimate and the score statistic. The tests based on the score statistics and $M$-estimates are put in a form such that calculations are the same as those for the test based on the mean. Two measures of asymptotic relative efficiency (ARE) are used for the comparison of the two tests. The ARE of Pitman is calculated for the two tests for the general robust function $\psi(x)$ under some conditions on it. The ARE of Pitman is equal to one. Bahadur slopes which give the ARE of Bahadur have been compared for the two statistics for Huber's scores, $b=1$. Neither of these tests has greater slope over the whole range. In Section 1 of Chapter 5, the one sample problem is considered. Section 2 considers the two sample situation. Both of them have the similar results.

\section{REFERENCES}

[1] S. Froda and C. van Eeden, 'A uniform saddlepoint expansion for the null-distribution of the Wilcoxon-Mann-Whitney statistic', Canad. J. Statist. 28 (2000), 137-149.

Integral Energy

PO Box 6366

Blacktown NSW 2148

Australia 